\title{
Determinants of the Duration of the Refractory Period of the Atrioventricular Nodal System in Man *
}

\author{
Joseph W. Linhart, Eugene Braunwald, $†$ and John Ross, Jr. \\ (From the Cardiology Branch, National Heart Institute, Bethesda, Md.)
}

One of the fundamental characteristics of nervous tissue, skeletal muscle, and myocardium is their refractoriness for short periods following depolarization (1). This period, during which a propagated action potential cannot be evoked by a stimulus, has been designated the "effective refractory period" (2), and the effective refractory period thus limits the maximal rate at which depolarizations can occur. When normal atrioventricular (AV) conduction takes place, the ventricular contraction rate is limited by the $\mathrm{AV}$ nodal system, which has been shown in experimental animals to have a longer effective refractory period than atrial or ventricular tissue (3). In spite of the fundamental importance of the refractory period of the AV nodal conduction system, this interval has heretofore been estimated only from electrocardiograms obtained from patients with spontaneous cardiac arrhythmias (4). The presence of arrhythmias may be associated with abnormalities of conduction, and the analysis of such electrocardiograms does not allow systematic investigation of the factors that influence this period in normal subjects. In the present investigation a practical method for the measurement of the effective refractory period of the $\mathrm{AV}$ nodal system in conscious human subjects is described. With this method normal basal values for this period were established, and the effects of tachycardia, exercise, atropine, and of various sympathomimetic drugs on its duration are presented.

\section{Methods}

A bipolar electrode catheter ${ }^{1}$ was passed into the right atrium and positioned against the mid-portion of the lateral wall of the right atrium. A slight bend was

\footnotetext{
* Submitted for publication October 13, 1964; accepted February 4, 1965.

$\dagger$ Address requests for reprints to Dr. Eugene Braunwald, National Heart Institute, Bethesda, Md. 20014.

1 NBIH catheter, United States Catheter \& Instrument Corp., Glens Falls, N. Y.
}

maintained near the tip of the catheter to insure adequate contact with the wall. The electrodes were connected to a stimulus isolation unit, and the leads contained a $10,000 \mathrm{ohm}$ resistor, making small changes in resistance at the site of stimulation of no practical significance and preventing large fluctuations of current. The stimulus isolation unit prohibited the accidental development of large electrical potentials between the stimulating circuit and the other equipment to which the patient was attached. The stimulator ${ }^{2}$ provided a square wave impulse of $6 \mathrm{msec}$ duration, and was adjusted to produce a current at the right atrial wall of 2.5 to $4.0 \mathrm{ma}$. The voltage setting of the stimulator ranged between 50 and $90 \mathrm{v}$ and was set at approximately twice the threshold level. All measurements were made on lead II of the standard surface electrocardiogram.

The term "refractory period of the atrioventricular nodal conduction system" (AVRP), as used in this paper is, as discussed below, closely analogous to the effective refractory period (2) and refers to the longest time interval between two stimuli delivered to the atrium, both of which evoke propagated atrial depolarizations, but the second of which fails to result in a propagated ventricular depolarization. The technique for measuring AVRP was adapted from that described by Krayer, Mandoki, and Mendez for use in the isolated heart-lung preparation (5). Unless specifically noted, all of the observations were carried out by the "extra-stimulus" method, which consisted of driving the atrium electrically at a basic regular rate just greater than the sinus rate, and delivering occasional single extra premature stimuli at specific time intervals following these basic driving stimuli. The longest time interval between a driving stimulus and a premature stimulus that did not evoke a propagated ventricular depolarization was taken as the AVRP. The technique is illustrated diagrammatically in Figure 1, lines $A$ and $B$, where $S_{1}$ to $S_{5}$ represent the basic driving stimuli. In line $A$, the extra stimulus, delivered between $S_{3}$ and $S_{4}$, initiates a $P$ wave that arrives at the AV nodal conduction system when it is no longer refractory, and a QRS complex results. The time interval between the driving and the premature stimuli was then shortened in $10 \mathrm{msec}$ steps until the premature impulse failed to evoke a $Q R S$ complex. In line $B$ the premature stimulus is delivered so early that the atrial depolarization wave arrives at a time when the AV nodal conduction system is still refractory, and no QRS com-

2 American Electronics Laboratories, model 104A, Colmar, $\mathrm{Pa}$. 


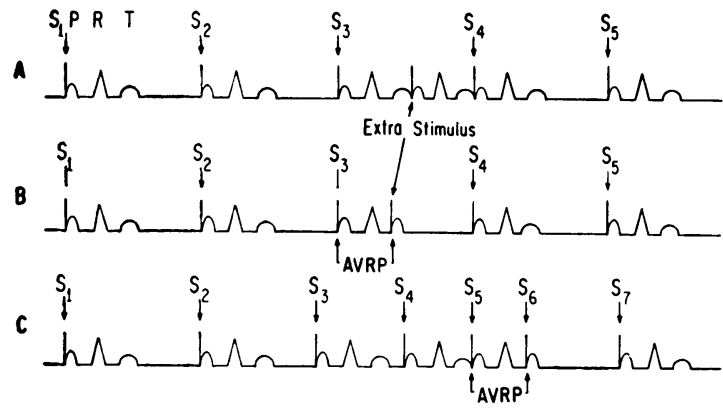

Fig. 1. Schematic illustration of the method emPLOYED FOR MEASURING THE REFRACTORY PERIOD OF THE atrioventricular nodal System (AVRP). For detailed explanation see text.

plex is evoked. Figure 2A shows an electrocardiogram in which the premature stimulus, introduced between $S_{2}$ and $S_{3}$, initiates a $P$ wave and a QRS complex (although the latter is delayed, and as a consequence $S_{3}$ is not conducted to the ventricles). In contrast, in the tracing shown in Figure $2 \mathrm{~B}$, the premature stimulus (between $\mathrm{S}_{3}$ and $\mathrm{S}_{4}$ ) is delivered $30 \mathrm{msec}$ earlier, the atrial depolarization wave reaches the AV conduction system during the AVRP, the impulse is not transmitted to the ventricles, and no $Q R S$ complex occurs. Since the intervals were shortened by steps of $10 \mathrm{msec}$, alterations in the AVRP of less than $10 \mathrm{msec}$ could not be detected by this technique.

After determination of the AVRP by the extra-stimulus method described above, the effects of one or more interventions on this measurement were studied. Whenever the effects of more than one intervention were determined in the same patient, a control measurement was repeated between the interventions. The effects of induced tachycardia on the AVRP were determined in nine patients by first making the measurement at a rate just greater than the sinus rate, and by repeating it 5 minutes after the rate had been elevated by the electrical pacemaker. The effects of the following drugs infused intravenously were studied: isoproterenol, 1.0 to $1.5 \mu \mathrm{g}$ per minute (seven patients) ; methoxamine, 0.95 to $1.40 \mathrm{mg}$ per minute (two patients); phenylephrine, 60 to $240 \mu \mathrm{g}$ per minute (six patients) ; norepinephrine, 1.6 to $3.2 \mu \mathrm{g}$ per minute (three patients) ; and atropine, $0.5 \mathrm{mg}$ to 1.0 $\mathrm{mg}$ as a single injection (11 patients). In two patients the effects of phenylephrine on AVRP were studied both before and after atropine. In seven patients the effects on the AVRP of leg exercise, consisting of pedaling a bicycle ergometer in the supine position at $40 \mathrm{rpm}$ at an external work load of approximately $500 \mathrm{ft}$-lbs per minute for 5 minutes, were determined.

In 15 patients the AVRP was also measured by a second technique, termed the "increasing rate" method, described in detail previously (6), and diagrammatically illustrated in Figure 1C. The basic rate of stimulation was gradually increased until an interval between stimuli was reached which was so short that a stimulus resulted in atrial depolarization but was not transmitted to the ventricles.

A total of 33 patients ranging in age from 11 to 59 years were studied in the postabsorptive state, 1 hour after the administration of $100 \mathrm{mg}$ sodium pentobarbital. None of the patients had atrioventricular or intraventricular conduction abnormalities, the P-R intervals and $Q R S$ durations in each patient being normal in the basal state. No patients had experienced congestive heart failure or had marked limitation of cardiac reserve at the time of the study. None of the patients were receiving digitalis, quinidine, or procaine amide, and the serum electrolytes were always within normal limits. Eleven of the patients had functional heart murmurs, six had mitral stenosis, five had atrial septal defects, and three had mild aortic stenosis. In one patient each the dignosis was ventricular septal defect, mild aortic regurgitation, idiopathic hypertrophic subaortic stenosis, right ventricular myxoma, patent ductus arteriosus, cardiomyopathy, status postclosure of a ventricular septal defect, and status postaortic valve replacement for aortic stenosis. In every instance the measurements of AVRP followed the diagnostic cardiac catheterization.

\section{Results}

I. Control values. The control values of the AVRP in 20 patients studied by the extra-stimulus method averaged $350 \pm 53 \mathrm{msec}(\mathrm{SD})$, a value which was significantly shorter $(p<0.01)$ than that obtained by the increasing rate method in 15 patients, which averaged $413 \pm 52 \mathrm{msec}$.

II. Tachycardia (Figure $3 A$ ). In nine patients the AVRP was first measured at a rate just above the spontaneous sinus rate, which ranged from 68 to 102 and averaged 84 per minute; at this rate the AVRP ranged between 250 and 430 and averaged $344 \mathrm{msec}$. The atrial rate was then increased to between 109 and 131 (average $=120$ per minute), and the AVRP rose significantly $(p<0.02)$ to between 340 and $450 \mathrm{msec}$ (average $=399 \mathrm{msec}$ ). Thus, abbreviation of the R-R interval by an average of $215 \mathrm{msec}$ (30\% of control) was associated with a prolongation of the AVRP by an average of $55 \mathrm{msec}(16 \%$ of control).

III. Atropine (Figure 3B). In 11 patients the heart rate before atropine ranged between 68 and 92 and averaged 77 per minute, while the AVRP ranged between 280 and 430 and averaged 344 msec. Atropine resulted in an elevation of heart rate to an average of 103 per minute. In contrast to the finding when heart rate was increased by 
electrical stimulation, AVRP declined significantly ( $\mathrm{p}<0.01$ ), falling to between 160 and $330 \mathrm{msec}$ ( average $=246 \mathrm{msec}$ ). Thus, an average fall of the R-R interval of $195 \mathrm{msec}$ ( $25 \%$ of control) was associated with a decline of the AVRP averaging $98 \mathrm{msec}$ ( $29 \%$ of control).

IV. Isoproterenol (Figure 3C). In seven patients the control heart rate averaged 82 per minute and rose to an average level of 95 per minute during the isoproterenol infusion. AVRP ranged between 300 and 500 and averaged $405 \mathrm{msec}$ during the control period, falling in each patient to an average value of $300 \mathrm{msec}$, which was significantly lower $(\mathrm{p}<0.01)$ than that noted during the control period. Thus, an average decrease in the $\mathrm{R}-\mathrm{R}$ interval of $90 \mathrm{msec}$ ( $12 \%$ of control) was
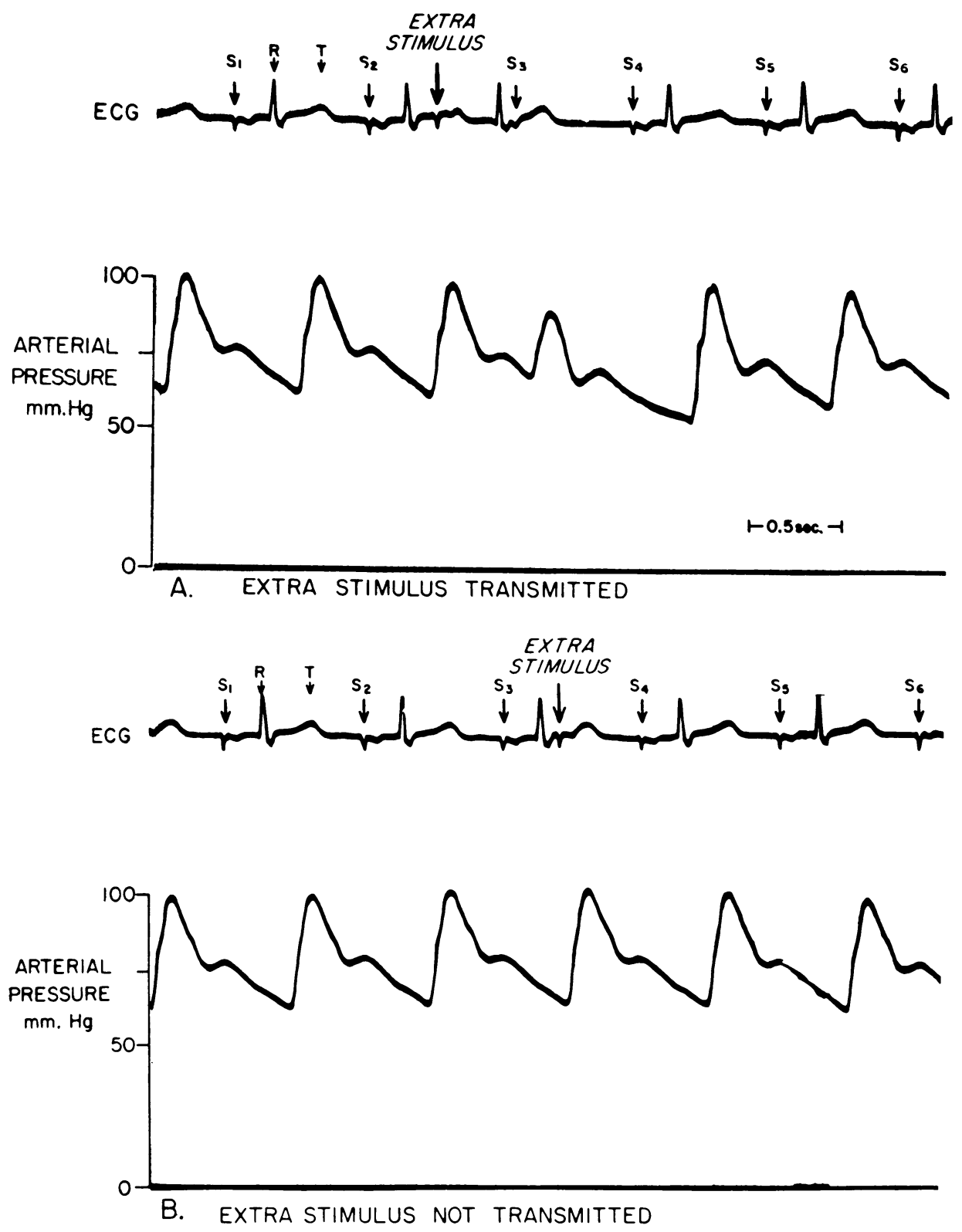

Fig. 2. Simultaneous recordings of Electrocardiogram AND ARTERIAL PRESSURE PUlse DURING DETERMINATION OF AVRP. $S_{1}$ to $S_{\varepsilon}$ represent electrical stimuli. In tracing A (top) the extra stimulus, delivered between $S_{2}$ and $S_{3}$, was transmitted to the ventricles, but in tracing $B$ (bottom) the extra stimulus, delivered between $S_{3}$ and $S_{4}$, was not transmitted. 

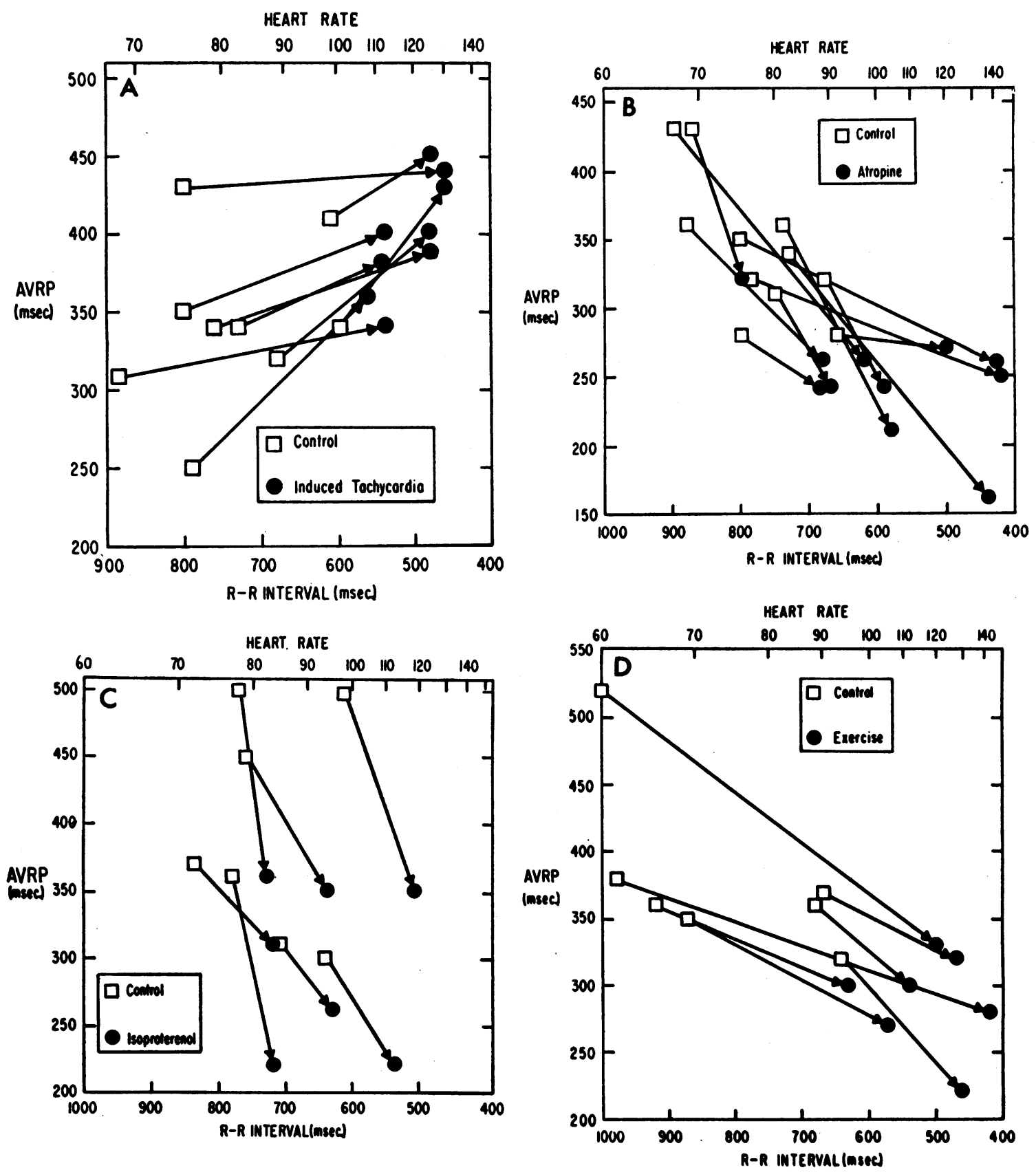

Fig. 3. EFFect of A) INDUCED TACHYCARDIA, B) ATROPINE, C) ISOPROTERENOL, AND D) MUSCULAR EXERCISE ON THE RELATIONSHIP BETWEEN THE AVRP AND THE R-R INTERVAL.

associated with an abbreviation of the AVRP, which averaged $105 \mathrm{msec}(26 \%$ of control).

V. Exercise (Figure 3D). In seven patients the heart rate at rest ranged between 60 and 95 and averaged 72 per minute, while the AVRP ranged between 320 and 520 , averaging $390 \mathrm{msec}$. Muscular exercise raised heart rate to a mean value of 117 per minute, while AVRP fell significantly $(p<0.01)$ to an average value of 280 msec. Thus, an average decline of the R-R interval of $320 \mathrm{msec}$ ( $28 \%$ of control) was associated with an abbreviation of the AVRP, which averaged $110 \mathrm{msec}$ (39\% of control).

VI. Phenylephrine and methoxamine (Figure 
$4 A$ ). The administration of phenylephrine and methoxamine, in doses sufficient to elevate the systolic pressure by an average of $56 \mathrm{~mm} \mathrm{Hg}$ and the diastolic pressure by $28 \mathrm{~mm} \mathrm{Hg}$, slowed heart rate, from an average value of 75 per minute during the control period to one of 56 per minute. This was associated with a significant prolongation of the AVRP from an average of 326 to 578 msec $(p<0.01)$. Thus, an average increase of the R-R interval of $274 \mathrm{msec}$ ( $34 \%$ of control) was associated with a lengthening of the AVRP, which averaged $252 \mathrm{msec}$ (77\% of control).

VII. Phenylephrine and atropine (Figure $4 B$ ). In two patients the effects of phenylephrine were first studied, as described above, and after a second control measurement atropine was administered. After redetermination of the AVRP phenylephrine was reinfused to produce an identical elevation of arterial pressure. In these two patients the individual effects of phenylephrine and atropine on both the AVRP and the R-R interval were similar to those already described (Figure $4 \mathrm{~A}$ and Figure 3B). However, when phenylephrine was reinfused after atropine, neither the AVRP nor the R-R interval was altered.

VIII. Norepinephrine (Figure $4 C$ ). The administration of norepinephrine to three patients slowed heart rate from an average of 78 per minute to 70 per minute while AVRP increased from an average value of 330 to $410 \mathrm{msec}$.

\section{Discussion}

Previous studies on the refractory period of the AV nodal conduction system have been carried out in a variety of experimental animal preparations. Krayer and collaborators showed that epinephrine shortens the functional refractory period of the AV nodal system in the isolated canine heart-lung preparation (5). Subsequently Mendez, Aceves, and Mendez found that pentobarbital and cardiac glycosides prolong this period and that at certain dose levels this action of the glycosides could be reduced by prior sympathetic denervation $(7,8)$.

Rosenblueth, Mendez, and their respective collaborators indicate that the functional refractory period of excitable tissue is the shortest attainable interval between two induced responses when the second stimulus is delivered at 2 or more times the threshold intensity $(1,3,9)$. They emphasized, however, that this value is attended by an error equal to any difference that might occur in the propagation time from the point of stimulation to the point of recording between the first and second responses. In the present investigation on man it was observed that this error was inconstant and frequently quite large. For example, in the tracing reproduced in Figure 2A, the ventricular depolarization resulting from the interposed stimulus was delayed by $120 \mathrm{msec}$. This potential error results primarily from variations in the conduction velocity in the $\mathrm{AV}$ conduction system, which can be altered by influences that also modify the duration of the AVRP. Accordingly, rather than determining the shortest period between two induced responses, we measured the longest interval between two stimuli that could be achieved when the second stimulus failed to evoke a propagated ventricular depolarization. This measurement conforms closely- to the "effective refractory period" defined by Hoffman and Cranefield (2), although the strength of the interposed stimulus in our study was not maximal but only twice the diastolic threshold level. Since the stimulus was applied to the atrium, rather than directly to the AV node, alterations of conduction velocity within the atrium would modify the duration of the AVRP. However, in the present investigation no detectable alterations in atrial conduction, as determined from the duration of the atrial depolarization wave, occurred.

In the interpretation of changes in the AVRP observed in the present investigation the complexities of AV conduction, which have recently been reviewed $(10,11)$, must be considered. Thus, many findings are consistent with the hypothesis that decremental conduction occurs in the $\mathrm{AV}$ conduction system (10), and recordings of transmembrane action potentials have shown that conduction is slowest across a narrow zone at the atrial margin of the AV node (12). In the isolated rabbit heart, Cranefield, Hoffman, and Paes de Carvalho have shown that the failure of $\mathrm{AV}$ transmission caused by acetylcholine takes place in fibers at the atrial margin of the node (13), whereas lower portions of the node appear to be involved in the failure of transmission caused by tachycardia (10).

Thus, although determination of the AVRP 


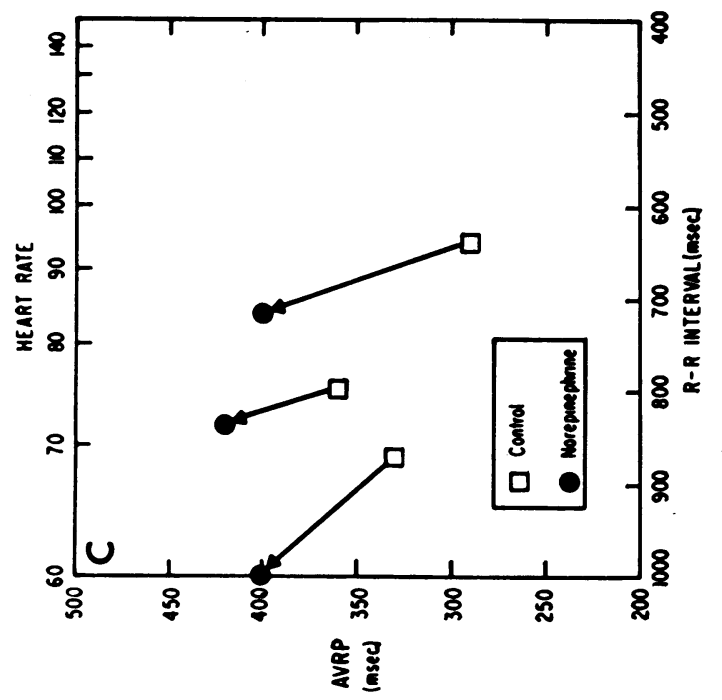

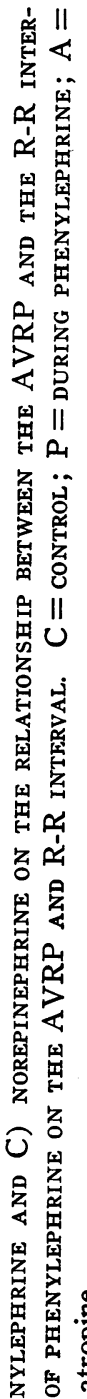
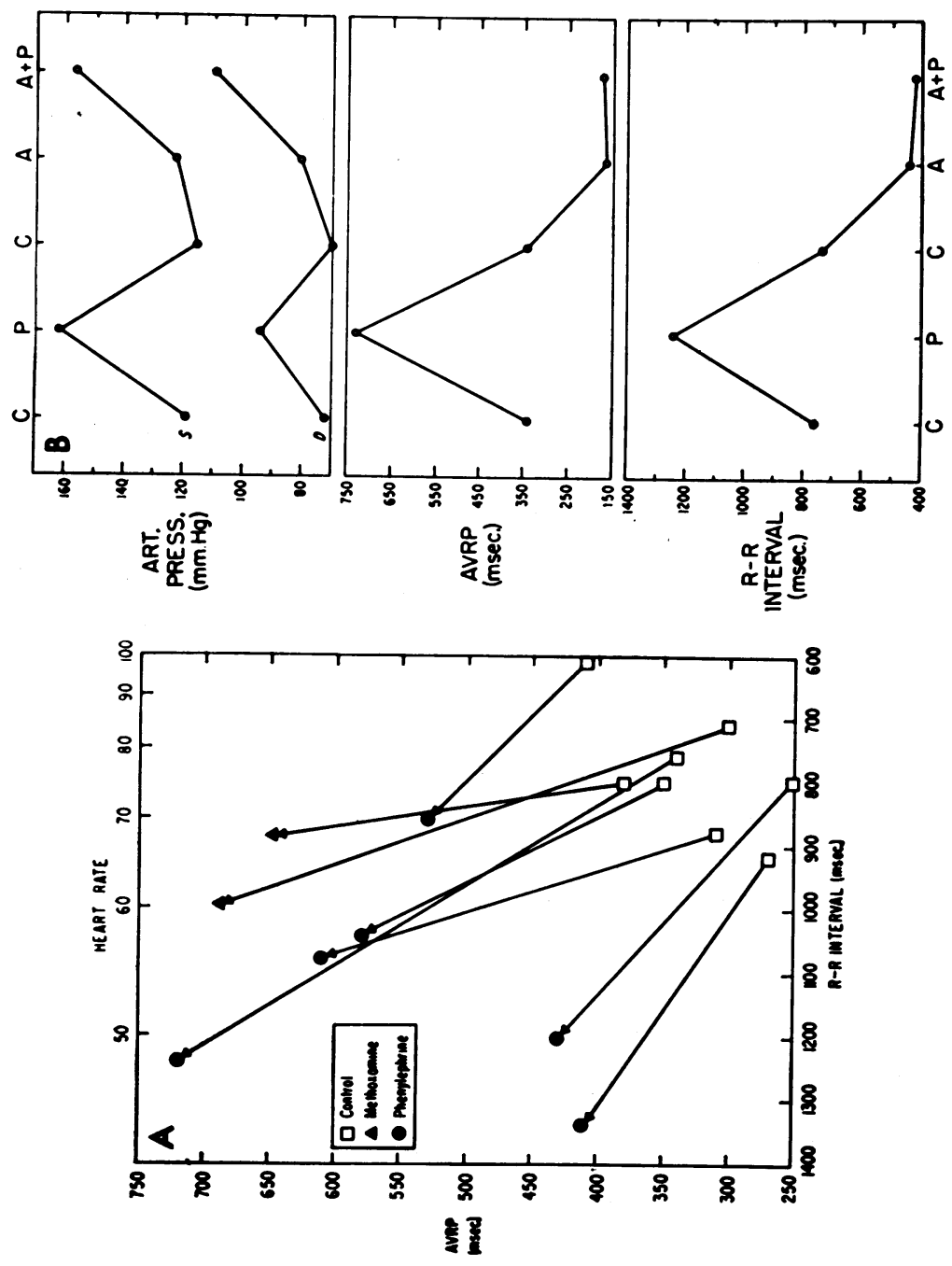

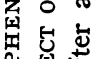

的票

究究

国

总客

我密

密

舆䍃影

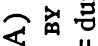

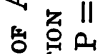

过 +

密 4

究兽范

的蓄

空宾离 
does not simply provide a measurement of the period during which the AV node is totally inexcitable or unresponsive, the measurement does provide an assessment of the time interval following a stimulus during which the AV nodal conduction system is incapable of transmitting an impulse that can initiate a propagated ventricular depolarization. From the present investigation in man it is apparent that this period is not a static one, but that many common interventions can profoundly modify its duration. Tachycardia, produced by electrical stimulation of the atrium, tended to lengthen the AVRP slightly, a finding that correlates with the longer duration of the AVRP when it is measured by the increasing rate than when it is determined by the extra-stimulus method. It has previously been observed in normal human subjects that when the rate of stimulation of the right atrium is increased the interval between the stimulus and the QRS increases (14). Although the results of the present study are opposite to those described by Mendez, Gruhzit, and Moe in the anesthetized, open-chest, acutely denervated $\operatorname{dog}$ heart (3), they are consistent with the observation of a reduced amplitude and rate of rise of the transmembrane potential in the atrial part of the AV node during rapid electrical stimulation of the atrium (10). Therefore the shortening of the AVRP that occurred when tachycardia was induced by the administration of atropine and isoproterenol or by muscular exercise is even more striking when it is considered that tachycardia per se tends to lengthen this interval.

Alterations in the activity of both the sympathetic and parasympathetic nervous systems affect both the AV conduction system and the sinoatrial node. However, the relationship between the R-R interval and the duration of the AVRP was not a linear one, and indeed, the relative changes in the duration of the AVRP induced by atropine, isoproterenol, phenylephrine, methoxamine, and norepinephrine were often greater than the simultaneous changes that occurred in the duration of the $\mathrm{R}-\mathrm{R}$ interval. The abbreviation of the AVRP associated with the administration of atropine and of isoproterenol is interpreted, respectively, as the effect of the withdrawal of parasympathetic influences from, and of the activation of adrenergic receptors in, the AV conduction system. Muscular exercise shortened the AVRP in a manner similar to atropine or isoproterenol, and it is likely that both stimulation of sympathetic receptors and reduction of parasympathetic inhibition were involved in the response. On the other hand, phenylephrine and methoxamine, two sympathomimetic pressor drugs that have no direct cardiac effects (15), simultaneously prolonged this period and slowed the sinus rate.

The finding in two patients that phenylephrine did not alter the AVRP after atropine supports the view that phenylephrine has no direct effect on the AV conduction system, although since only two patients were studied these results cannot be considered conclusive. It is well known that the slowing of heart rate produced by these drugs is reflexly mediated, and it has been shown, both in the dog and in man, that the efferent limb of the reflex to the sinoatrial node that is stimulated by elevating arterial pressure, is mediated only by the parasympathetic system (16). The finding that atropine completely prevented the prolongation of the AVRP induced by phenylephrine (Figure 4B) indicates that as a consequence of baroreceptor stimulation, the AV conduction system, like the sinoatrial node, is inhibited only by parasympathetic stimulation, withdrawal of tonic sympathetic activity playing no detectable role. Norepinephrine, like isoproterenol, stimulates adrenergic receptors in the heart, and it might therefore be expected to abbreviate the AVRP. However, the prolongation of the AVRP that occurred with norepinephrine was probably reflex in nature, consequent to its pressor action, and this effect probably masked any direct action of the catecholamine. Since only three patients received norepinephrine, however, these results must be considered to be tentative.

The chief finding that emerges from this study is that the AV conduction system in conscious human subjects is affected by many influences, both direct and reflex. In general, the effects of any given stimulus on the rhythmicity of the sinoatrial node (as reflected in the R-R interval) are directionally similar to those on the AV system (as reflected in the AVRP), although in many instances the relative effect on the latter is greater than on the former. Under most clinical circumstances, effects on the sinoatrial node, since they alter ventricular rate, are more obvious than effects on the AV system. Nevertheless, the 
AVRP is of considerable clinical importance, since it limits the ventricular rate in patients with a variety of supraventricular tachycardias, including atrial fibrillation. Furthermore, the delay in conduction imposed by the AV system regulates the time interval between the contractions of the atria and ventricles and thereby plays a fundamental role in regulating the atrial contribution to ventricular filling (17).

\section{Summary}

A technique was devised for measurement of the refractory period of the atrioventricular conduction system (AVRP) in man. A bipolar electrode catheter was placed against the right atrial wall and stimulated the right atrium at a constant rate just above the sinus rate. Extra impulses were interposed between the regular stimuli; the time intervals between these extra impulses and the preceding stimuli were then shortened progressively. The time interval at which AV conduction just failed was taken as the AVRP. In 20 unanesthetized patients, studied in the basal state, the AVRP averaged $350 \mathrm{msec}$. In nine patients, atrial tachycardia, induced by rapid electrical stimulation of the right atrium, prolonged the AVRP. In seven patients muscular exercise shortened the AVRP by an average of $10 \mathrm{msec}$. Isoproterenol (seven patients) and atropine (11 patients) resulted in reductions of the AVRP similar to those observed during exercise. Elevations of arterial pressure with methoxamine or phenylephrine in eight patients prolonged AVRP by an average of $252 \mathrm{msec}$. Since this prolongation could be prevented by atropine, it was considered to result from reflex vagal stimulation. Thus, in any given patient the AVRP is affected profoundly by many influences, including sympathetic and parasympathetic stimuli.

\section{References}

1. Rosenblueth, A., J. Alanís, and J. Mandoki. The functional refractory period of axons. J. cell. comp. Physiol. 1949, 33, 405.

2. Hoffman, B. F., and P. F. Cranefield. Electrophysiology of the Heart. New York, McGraw-Hill, 1960 , p. 253.

3. Mendez, C., C. C. Gruhzit, and G. K. Moe. Influence of cycle length upon refractory period of auricles, ventricles, and $\mathrm{A}-\mathrm{V}$ node in the dog. Amer. J. Physiol. 1956, 184, 287.

4. Katz, L. N., and A. Pick. Clinical Electrocardiography. I. The Arrhythmias. Philadelphia, Lea \& Febiger, 1956, p. 30.

5. Krayer, O., J. J. Mandoki, and C. Mendez. Studies on veratrum alkaloids XVI. The action of epinephrine and of veratramine on the functional refractory period of the auriculo-ventricular transmission in the heart-lung preparation of the dog. J. Pharmacol. exp. Ther. 1951, 103, 412.

6. Morrow, D. H., T. E. Gaffney, and E. Braunwald. Studies on digitalis VIII: Effects of autonomic innervation and of myocardial catecholamine stores upon the cardiac action of ouabain. J. Pharmacol. exp. Ther. 1963, 140, 236.

7. Méndez, R., and C. Méndez. The action of cardiac glycosides on the refractory period of heart tissues. J. Pharmacol. exp. Ther. 1953, 107, 24.

8. Mendez, C., J. Aceves, and R. Mendez. The antiadrenergic action of digitalis on the refractory period of the A-V transmission system. J. Pharmacol. exp. Ther. 1961, 131, 199.

9. Rosenblueth, A. Functional refractory period of cardiac tissues. Amer. J. Physiol. 1958, 194, 171.

10. Hoffman, B. F., and P. F. Cranefield. Electrophysiology of the Heart. New York, McGraw-Hill, 1960, pp. 132-175.

11. Scher, A. M. Excitation of the heart in Handbook of Physiology, Section 2, Circulation, W. F. Hamilton and P. Dow, Eds. Washington, D. C., American Physiological Society, 1962, pp. 294-298.

12. Hoffman, B. F., A. Paes de Carvalho, W. C. Mello, and P. F. Cranefield. Electrical activity of single fibers of the atrioventricular node. Circulat. Res. 1959, 7, 11.

13. Cranefield, P. F., B. F. Hoffman, and A. Paes de Carvalho. Effects of acetylcholine on single fibers of the atrioventricular node. Circulat. Res. 1959, 7, 19.

14. Warner, H. F., and D. H. Lewis. Effect of increased pulse rate on circulatory dynamics in normal resting man. Fed. Proc. 1961, 20, 131.

15. Goldberg, L. I., R. D. Bloodwell, E. Braunwald, and A. G. Morrow. The direct effects of norepinephrine, epinephrine, and methoxamine on myocardial contractile force in man. Circulation 1960, 22, 1125.

16. Glick, G., and E. Braunwald. The relative roles of the sympathetic and parasympathetic nervous systems in the reflex control of heart rate. Circulat. Res. 1965, 16, 363.

17. Braunwald, E., and C. J. Frahm. Studies on Starling's law of the heart. IV: Observations on the hemodynamic functions of the left atrium in man. Circulation 1961. 24. 633. 